\title{
CONSTRUCTION OF MEASURES AND INTEGRALS
}

\author{
BY \\ B. S. THOMSON
}

\begin{abstract}
The systems introduced by R. Henstock and later by E. J. McShane to provide powerful generalizations of the Riemann integral are used to construct outer measures and upper integrals and to develop a Lebesgue type theory in quite general settings.
\end{abstract}

In this paper we present an introduction to the theory of measure and integration in division spaces. These spaces were first introduced by Henstock [3] in order to generalize the classical Riemann integral to a setting which permitted limit theorems of Lebesgue power; most of the results are essentially due to Henstock. The presentation here is concerned only with the construction of measures and of a Lebesgue-type integral. For the Riemann theory which permits a definition of the integral for functions with values in a topological semigroup and for the generalized limit theorems see Henstock [4] and McShane [5].

1. Division systems. Let $T$ be a set, $\mathfrak{B}$ a semi-clan (semiring) of subsets of $T$, and $\mathbb{E}$ the clan (ring) generated by $\mathfrak{B}$. The elements of $\mathbb{E}$ will be called the elementary sets and the pairs in $\mathfrak{B} \times T$ will be called bricks. Any finite subset $\mathscr{D}$ of $\mathfrak{B} \times T$ will be called a division provided the collection of $I \in \mathfrak{B}$ with $(I, x) \in \mathfrak{D}$ for some $x \in T$ is disjointed. We write $\sigma(\mathfrak{D})=\bigcup\{I:(I, x) \in \mathfrak{D}\}$ for any division $\mathfrak{D}$ and call $\mathfrak{D}$ a division of the elementary set $E$ where $E=\sigma(\mathfrak{D})$. Also a subset $S$ of $\mathfrak{B} \times T$ is said to divide $E$ if $\boldsymbol{S}$ contains a division of $E$.

For any subset $S$ of $\mathfrak{B} \times T$ and any family $\mathfrak{A}$ of subsets of $\mathfrak{B} \times T$ we shall require a notation for the following collections relative to a subset $X \subseteq T$.

(1.1) $S[X]=\{(I, x) \in S: x \in X\}$,

(1.2) $\mathfrak{A}[X]=\{\boldsymbol{S}[X] ; \boldsymbol{S} \in \mathfrak{A}\}$,

(1.3) $S(X)=\{(I, x) ; I \subseteq X\}$,

(1.4) $\mathfrak{A}(X)=\{\boldsymbol{S}(X): S \in \mathfrak{A}\}$.

Definition 1. An ordered triple $(T, \mathfrak{A}, \mathfrak{B})$ is said to be a division system if $\mathfrak{A}$ is a collection of subsets of $\mathfrak{B} \times T$ such that

(i) $(\varnothing, x) \in S$ for every $S \in \mathfrak{A}$ and every $x \in T$.

(ii) $\mathfrak{A}$ is directed downwards by set inclusion.

A division system $(T, \mathfrak{A}, \mathfrak{B})$ is said to be fully decomposable (resp. decomposable)

Received by the editors May 18, 1970.

AMS 1970 subject classifications. Primary 28A10, 28A25.

Key words and phrases. Variation, outer measure, upper integral, Lebesgue integral, Lebesgue space.

Copyright (C) 1971, American Mathematical Society 
if for every family (resp. countable family) $\left\{X_{i}: i \in I\right\}$ of disjoint subsets of $T$ and every $\left\{S_{i}: i \in I\right\} \subseteq \mathfrak{A}$ there exists an $S$ in $\mathfrak{A}$ with $S\left[X_{i}\right] \subseteq S_{i}\left[X_{i}\right](i \in I)$.

Any real-valued function $\mu$ on $\mathfrak{B} \times T$ will be called a brick function; a brick function $\mu$ which has the property that $I \rightarrow \mu(I, x)$ is additive on the semi-clan $\mathfrak{B}$ for every fixed $x \in T$ will be called an additive brick function. In particular if $m$ is an additive function on $\mathfrak{B}$ and $f$ is a function on $T$ the function $f m:(I, x) \rightarrow f(x) m(I)$ is certainly an additive brick function. If $\mathfrak{D} \subseteq \mathfrak{B} \times T$ is a division we shall write (D) $\sum \mu(I, x)$ for the sum $\sum\{\mu(I, x):(I, x) \in \mathscr{D}\}$, replacing an empty sum by zero.

Let $S$ be an arbitrary subset of $\mathfrak{B} \times T$ and let $\mathfrak{A}$ be an arbitrary family of such subsets. Then for a brick function $\mu$ we have the following concepts for the variation of $\mu$ relative to $S$ and $\mathfrak{A}$.

(1.5) $V(\mu, S)=\sup \left\{(\mathscr{D}) \sum|\mu(I, x)|: \mathscr{D} \subseteq S, \mathfrak{D}\right.$ a division $\}$.

(1.6) $V(\mu, \mathfrak{A})=\inf \{V(\mu, \boldsymbol{S}): S \in \mathfrak{A}\}$.

THEOREM 1.7. Let $\mu$ be a brick function on a decomposable division system $(T, \mathfrak{A}, \mathfrak{B})$ and denote $\mu^{*}(X)=V(\mu, \mathfrak{A}[X])(X \subseteq T)$. Then

(i) $\mu^{*}(\varnothing)=0,0 \leqq \mu^{*}(X) \leqq+\infty$, and

(ii) $\mu^{*}(X) \leqq \sum_{j=1}^{\infty} \mu^{*}\left(X_{j}\right)\left(X \subseteq \bigcup_{j=1}^{\infty} X_{j}\right)$.

Proof. In standard terminology this theorem asserts that $\mu^{*}$ is a measure (or outer measure) on $T$. Assertion (i) is trivial. For (ii) let $\varepsilon>0$ and choose $S_{j} \in \mathfrak{A}$ so that $V\left(\mu, S_{j}\left[X_{j}\right]\right) \leqq \mu^{*}\left(X_{j}\right)+\varepsilon / 2^{j}$ for each integer $j$.

By the decomposable property choose $S \in \mathfrak{A}$ such that $S\left[X_{j} \backslash \hat{X}_{j-1}\right] \subseteq S_{j}\left[X_{j} \backslash \hat{X}_{j-1}\right]$ for each $j$, where $\hat{X}_{k}=\bigcup_{j \leqq k} X_{j}$.

Then if $\mathfrak{D} \subseteq S[X]$ is a division write $\mathfrak{D}_{j}=\mathfrak{D}\left[X_{j} \backslash \hat{X}_{j-1}\right]$ so that

$$
\text { (D) } \begin{aligned}
\sum|\mu(I, x)| & =\sum_{j=1}^{\infty}\left(\mathfrak{D}_{j}\right) \sum|\mu(I, x)| \\
& \leqq \sum_{j=1}^{\infty} V\left(\mu, S_{j}\left[X_{j}\right]\right) \leqq \sum_{j=1}^{\infty} \mu^{*}\left(X_{j}\right)+\varepsilon .
\end{aligned}
$$

Thus $\mu^{*}(X) \leqq V(\mu, S[X]) \leqq \sum_{j=1}^{\infty} \mu^{*}\left(X_{j}\right)+\varepsilon$ and since $\varepsilon>0$ is arbitrary the result follows.

This method of constructing measures is apparently not widely known and is due to Henstock. Division systems as we have defined them are quite general: the necessity for $\mathfrak{B}$ to be a semi-clan can always be arranged by taking $\mathfrak{B}$ as the collection of all subsets of $T$. It is in the following definition and in particular in $\S 3$ below that this requirement assumes force.

Definition 2. A division system $(T, \mathfrak{A}, \mathfrak{B})$ is said to be of type $\mathrm{A}$ or type $\mathrm{B}$ if the corresponding property holds:

A. For every $\boldsymbol{S} \in \mathfrak{A}$ and every $J \in \mathfrak{B}$ if $(I, x) \in S$ then $(I \cap J, x) \in S$.

B. For every $J \in \mathfrak{B}$ there exists an $\boldsymbol{S} \in \mathfrak{A}$ such that if $(I, x) \in \boldsymbol{S}$ there is a finite disjointed sequence $I_{1}, I_{2}, \ldots, I_{n}$ in $\mathfrak{B}$ such that $I=\bigcup_{k=1}^{n} I_{k}$ and each $\left(I_{k} \cap J, x\right) \in S$ and $\left(I_{k} \backslash J, x\right) \in S$. 
Every division system of type A is evidently of type B. Certain properties of spaces of type A have been investigated by McShane [7] while Henstock has essentially used condition B in his investigations of the theory in Euclidean spaces. The principal result which we obtain now (Theorem 1.13) is that for these types with $\mu$ an additive brick function the measure $\mu^{*}$ has a property which usually requires a "regularity" assumption.

LeMMA 1.8. Let $\mu$ be an additive brick function on a division $\operatorname{system}(T, \mathfrak{Q}, \mathfrak{B})$ of type B. Then $V(\mu, \mathfrak{I})=V(\mu, \mathfrak{l}(E))+V(\mu, \mathfrak{I}(\mid E))(E \in \mathfrak{E})$.

Proof. Suppose that $E=J_{1} \cup J_{2} \cup \cdots \cup J_{k}$ for a disjointed sequence $\left\{J_{i}\right\}$ from $\mathfrak{B}$, and let $\boldsymbol{S}_{i} \in \mathfrak{A}$ be chosen by the hypothesis B relative to $J_{i} \in \mathfrak{B}$. Let $\boldsymbol{S} \in \mathfrak{A}$ such that $S \subseteq \bigcap_{i=1}^{k} \boldsymbol{S}_{k}$; then if $\mathfrak{D} \subseteq \boldsymbol{S}$ is a division and $(I, x) \in \mathcal{D}$ there is a disjointed sequence $I_{1}, I_{2}, \ldots, I_{n}$ from $\mathfrak{B}$ with $I=I_{1} \cup I_{2} \cup \cdots \cup I_{n}$ and each $\left(I_{k} \cap J_{1}, x\right)$, $\left(I_{k} \backslash J_{1}, x\right)$ belongs to $S$; applying the arguments again to each one of these latter bricks relative to $J_{2}, J_{3}$ etc. in turn we get (inductively) a disjointed sequence $I_{1}^{\prime}, I_{2}^{\prime}, \ldots, I_{m}^{\prime}$ with $I=I_{1}^{\prime} \cup I_{2}^{\prime} \cup \cdots \cup I_{m}^{\prime}$ and each $\left(I_{j}^{\prime} \cap E, x\right),\left(I_{j}^{\prime} \backslash E, x\right)$ belongs to $S$.

Since $\mu$ is additive then

$$
|\mu(I, x)| \leqq \sum_{j=1}^{m}\left|\mu\left(I_{j}^{\prime} \cap E, x\right)\right|+\left|\mu\left(I_{j}^{\prime} \mid E, x\right)\right| .
$$

If we apply this argument to each $(I, x) \in \mathcal{D}$ we obtain

$$
(D) \sum|\mu(I, x)| \leqq V(\mu, S(E))+V(\mu, S(\mid E))
$$

so that $V(\mu, S)=V(\mu, S(E))+V(\mu, S(\backslash E))$. As this holds for every $S \subseteq \bigcap_{i=1}^{k} S_{i}$ the lemma follows.

COROLlary 1.9. If $\mu$ is an additive brick function on a division $\operatorname{system}(T, \mathfrak{A}, \mathfrak{B})$ of type $\mathrm{B}$ then $E \rightarrow V(\mu, \mathfrak{A}(E))$ is additive on $\mathfrak{E}$.

Proof. If $E_{1}$ and $E_{2}$ are disjoint elementary sets we apply the lemma to the division system $(T, \mathfrak{A}(E), \mathfrak{B}) \quad\left(E=E_{1} \cup E_{2}\right)$ to get $V(\mu, \mathfrak{A}(E))=V\left(\mu, \mathfrak{A}\left(E_{1}\right)\right)$ $+V\left(\mu, \mathfrak{A}\left(E \backslash E_{1}\right)\right)$ and so $E \rightarrow V(\mu, \mathfrak{A}(E))$ is additive on $\mathfrak{E}$.

LEMMA 1.10. Let $\mu$ be an additive brick function on a division system of type B. If $V(\mu, S) \leqq V(\mu, \mathfrak{A})+\varepsilon<+\infty$ for some $\boldsymbol{S} \in \mathfrak{A}$ and $\varepsilon>0$ then $V(\mu, \boldsymbol{S}(E)) \leqq V(\mu, \mathfrak{R}(E))$ $+\varepsilon$ for every $E \in \mathbb{E}$.

Proof (Henstock [3, p. 231]). For any $\boldsymbol{S} \in \mathfrak{A}$

$$
V(\mu, \boldsymbol{S}(E)) \leqq V(\mu, S)-V(\mu, \boldsymbol{S}(\mid E)) \leqq V(\mu, \mathfrak{A})+\varepsilon-V(\mu, \mathfrak{A}(\mid E))
$$

and Lemma 1.8 completes the proof.

Corollary 1.11. Let $\mu$ be an additive brick function on a division system of type B. If $V(\mu, \mathfrak{A}(E))=0$ for every $E \in \mathfrak{E}$ and $V(\mu, \mathfrak{P})<+\infty$ then $V(\mu, \mathfrak{A})=0$. 
Proof. This follows directly from the lemma.

COROLlaRY 1.12. Let $\mu$ be an additive brick function on a division system of type B. If $V(\mu, A)<+\infty$ then $V(\mu, \mathfrak{A})=\sup _{E \in \mathfrak{E}} V(\mu, \mathfrak{A}(E))$.

Proof. If $\varepsilon>0$ choose $S \in \mathfrak{A}$ so that $V(\mu, \mathfrak{A})+\varepsilon \geqq V(\mu, S)$ and let $\mathfrak{D} \subseteq S(\sigma(\mathfrak{D})=E)$ so that (D) $\sum|\mu(I, x)| \geqq V(\mu, S)-\varepsilon$ and thus using the lemma

$$
\begin{aligned}
V(\mu, \mathfrak{A}(E))+\varepsilon & \geqq V(\mu, \boldsymbol{S}(E)) \geqq(\mathfrak{D}) \sum|\mu(I, x)| \\
& \geqq V(\mu, S)-\varepsilon \geqq V(\mu, \mathfrak{A})-\varepsilon
\end{aligned}
$$

and the result follows.

THEOREM 1.13. Let $\mu$ be an additive brick function on a decomposable division system of type B. Then for every increasing sequence $X_{1} \subseteq X_{2} \subseteq \cdots$ of subsets of $T$

$$
\mu^{*}\left(\bigcup_{k=1}^{\infty} X_{k}\right)=\lim _{k \rightarrow \infty} \mu^{*}\left(X_{k}\right)
$$

Proof (Henstock [3, p. 231]). Let $\varepsilon>0$, choose $\boldsymbol{S}_{k} \in \mathfrak{A}$ so that $V\left(\mu, \boldsymbol{S}_{k}\left[X_{k}\right]\right)$ $\leqq \mu^{*}\left(X_{k}\right)+\varepsilon / 2^{k}$ for each integer $k$ and then, by decomposability, choose $\boldsymbol{S} \in \mathfrak{A}$ so that $S\left[X_{k} \backslash X_{k-1}\right] \subseteq S_{k}\left[X_{k} \backslash X_{k-1}\right]$ (where $X_{0}=\varnothing$ ).

If $\mathfrak{D} \subseteq S\left[\bigcup_{k=1}^{\infty} X_{k}\right]$ is an arbitrary division write $\mathfrak{D}_{k}=\mathfrak{D}\left[X_{k} \mid X_{k-1}\right]$ and $E_{k}=\sigma\left(\mathfrak{D}_{k}\right)$, and let $m$ be the least integer for which $\mathfrak{D}_{k}=\varnothing(k>m)$.

Define $\mu_{k}(I, x)=\mu(I, x)$ if $x \in X_{k}$ and zero otherwise; then $\mu_{k}$ is also an additive brick function.

Now using 1.10, 1.9 and the definition of the $S_{k}$ we compute

$$
\text { (D) } \begin{aligned}
\sum|\mu(I, x)| & =\sum_{k=1}^{m}\left(\mathfrak{D}_{k}\right) \sum|\mu(I, x)| \leqq \sum_{k=1}^{m} V\left(\mu_{k}, S_{k}\left(E_{k}\right)\right) \\
& \leqq \sum_{k=1}^{m} V\left(\mu_{k}, \mathfrak{A}\left(E_{k}\right)\right)+\varepsilon \leqq \sum_{k=1}^{m} V\left(\mu_{m}, \mathfrak{l}\left(E_{k}\right)\right)+\varepsilon \\
& \leqq V\left(\mu_{m}, \mathfrak{X}\right)+\varepsilon \leqq \mu^{*}\left(X_{m}\right)+\varepsilon
\end{aligned}
$$

Hence

$$
\mu^{*}\left(\bigcup_{k=1}^{\infty} X_{k}\right) \leqq V\left(\mu, S\left[\bigcup_{k=1}^{\infty} X_{k}\right]\right) \leqq \lim _{k \rightarrow \infty} \mu^{*}\left(X_{k}\right)+\varepsilon
$$

This result together with 1.7 proves the theorem.

We introduce now a concept which provides a link between the values of the measure $\mu^{*}$ and the values of the brick function $\mu$. In particular if $m$ is an additive function on $\mathfrak{B}$ the function in the literature which usually serves as the variation of $m$ is defined on $E$ by $v(m, E)=V(m, \mathfrak{A}(E))$ (cf. Dunford and Schwartz [1, p. 97]) and the concept in Definition 3 provides a condition sufficient to ensure that $\mu^{*}(E)=v(m, E)$. 
Definition 3. Let $\mu$ be a brick function on a division system $(T, \mathfrak{A}, \mathfrak{B})$ and let $X \subseteq T$.

(i) $\mu$ is said to be regular from above at $X$ if $V(\mu, \mathfrak{P}[X](\backslash X))=0$.

(ii) $\mu$ is said to be regular from below at $X$ if $V(\mu, \mathfrak{A}[\backslash X](X))=0$.

(iii) $\mu$ is said to be regular at $X$ if both (i) and (ii) are true.

Among the immediate consequences of the definition we note the following: $\mu$ is regular from above at $X$ if and only if $\mu$ is regular from below at $\backslash X ; \mu$ is always regular from above at the empty set $\varnothing$ and is regular from below at $\varnothing$ if and only if $\mu(\varnothing, x)=0$ for every $x \in T$; the collection of sets at which $\mu$ is regular is closed under finite unions and intersections and under complements; if in addition the system is decomposable this is true for countable unions and intersections.

The importance of the concept lies in the following theorem.

THEOREM 1.14. Let $\mu$ be an additive brick function on a division system of type B. Then if $\mu$ is regular at an elementary set $E$

(i) $E$ is $\mu^{*}$-measurable, and

(ii) $\mu^{*}(E)=V(\mu, \mathfrak{U}(E))$.

Proof. If $\mu$ is regular at an elementary set $E$ then the relations

$$
V(\mu, \mathfrak{A}(E)[E]) \leqq V(\mu, \mathfrak{A}(E)) \leqq V(\mu, \mathfrak{A}[E](E))+V(\mu, \mathfrak{A}[\mid E](E))
$$

and

$$
V(\mu, \mathfrak{2}[E])=V(\mu, \mathfrak{A}[E](E))+V(\mu, \mathfrak{A}[E](\mid E))
$$

imply that $\mu^{*}(E)=V(\mu, \mathfrak{A}(E))$ as required for (ii).

Similarly it follows that $\mu^{*}(\mid E)=V(\mu, \mathfrak{2}(\mid E))$. Let $X \subseteq T$ be arbitrary and write $\mu_{0}(I, x)=\mu(I, x)$ if $x \in X$ and zero otherwise. Then if $\mu$ is additive and regular at $E$ so also is $\mu_{0}$.

Thus, using 1.8 and the above results,

$$
\begin{aligned}
\mu^{*}(X)=V\left(\mu_{0}, \mathfrak{A}\right) & =V\left(\mu_{0}, \mathfrak{A}(E)\right)+V\left(\mu_{0}, \mathfrak{A}(\backslash E)\right) \\
& =\mu_{0}^{*}(E)+\mu_{0}^{*}(\mid E)=\mu^{*}(X \cap E)+\mu^{*}(X \backslash E)
\end{aligned}
$$

so that $E$ is $\mu^{*}$-measurable as required.

We complete this section by stating a concept which is useful in applications of the above theory.

Definition 4. A division system $(T, \mathfrak{A}, \mathfrak{B})$ is said to be compatible with a topology $\tau$ on $T$ if for every open set $G$ in $\tau$ there is an $S \in \mathfrak{A}$ such that $S[G] \subseteq S(G)$.

It should be noted that the division system on the real line associated with the classical Riemann integral is not compatible with the usual topology on the real line but that the system in [2, p. 21] is. We mention the following fact as well: if $(T, \mathfrak{A}, \mathfrak{B})$ is a decomposable division system which is compatible with a topology on $T$ given by a metric $d$ then for any pair of sets $A, B \subseteq T$ if $d(A, B)>0$ then $\mu^{*}(A \cup B)=\mu^{*}(A)+\mu^{*}(B)$, so that $\mu^{*}$ is a metric outer measure on $T$. 
2. The upper integral. If $\mu$ is a brick function on a division system there is a natural way of defining an upper integral on $T$ relative to $\mu$. An upper integral is a function $N_{\mu}: \overline{\boldsymbol{R}}_{+}^{T} \rightarrow \overline{\boldsymbol{R}}_{+}$(where $\overline{\boldsymbol{R}}_{+}$denotes the nonnegative extended real numbers and $\overline{\boldsymbol{R}}_{+}^{T}$ the class of all functions $f: T \rightarrow \overline{\boldsymbol{R}}_{+}$) which satisfies certain of the properties expressed in Theorem 2.1 below.

Definition 5. If $\mu$ is a brick function on a division system $(T, \mathfrak{N}, \mathfrak{B})$ and if $f \in \overline{\boldsymbol{R}}_{+}^{T}$ we define

$$
N_{\mu}(f)=\sup _{m} V\left(f_{m} \mu, \mathfrak{A}\right)
$$

where $f_{m}(x)=f(x)$ if $f(x)<+\infty$ and $f_{m}(x)=m$ if $f(x)=+\infty$ (and as usual $f_{m} \mu$ is the brick function $\left.(I, x) \rightarrow f_{m}(x) \mu(I, x)\right)$.

THEOREM 2.1. Let $\mu$ be an additive brick function on a decomposable division system of type $\mathrm{B}$. Then

(1) $N_{\mu}(f)=0$ if and only if $f(x)=0$ a.e. $-\mu^{*}$.

(2) $N_{\mu}(f)<+\infty$ implies that $f(x)<+\infty$ a.e. $-\mu^{*}$.

(3) $N_{\mu}(\lambda f)=\lambda N_{\mu}(f)$ for every $\lambda>0$.

(4) If $f(x) \leqq \sum_{k=1}^{\infty} g_{k}(x)$ then $N_{\mu}(f) \leqq \sum_{k=1}^{\infty} N_{\mu}\left(g_{k}\right)$.

(5) If $f_{1}(x) \leqq f_{2}(x) \leqq f_{3}(x) \leqq \cdots$ and $f(x)=\sup _{k} f_{k}(x)$ then $N_{\mu}(f)=\sup _{k} N_{\mu}\left(f_{k}\right)$.

Proof. (1) If $X_{k}=\{x: f(x) \geqq 1 / k\}$ then $\mu^{*}\left(X_{k}\right) \leqq k N_{\mu}(f)=0$ so that setting $X_{0}=\{x: f(x) \neq 0\}=\bigcup_{k=1}^{\infty} X_{k}$ we have $\mu^{*}\left(X_{0}\right) \leqq \sum_{k=1}^{\infty} \mu^{*}\left(X_{k}\right)=0$ as stated.

(2) If $X_{\infty}=\{x: f(x)=+\infty\}$ then using the notation of Definition 5 we have $\mu^{*}\left(X_{\infty}\right) \leqq m^{-1} V\left(f_{m} \mu, \mathfrak{A}\right) \leqq m^{-1} N_{\mu}(f)$ for every $m$ and so $\mu^{*}\left(X_{\infty}\right)=0$ as stated.

(3) is trivial.

(4) Let $f_{m}(x)$ be defined as in Definition 5 and for each $x \in T$ and each integer $m$ let $n(x, m)$ be the least integer for which $((m-1) / m) f_{m}(x) \leqq \sum_{k=1}^{n(x, m)} g_{k}(x)$. Set $X_{n m}=\{x: n(x, m) \leqq n\}$ and then

$$
((m-1) / m) V\left(f_{m} \mu, \mathfrak{2}\left[X_{n m}\right]\right) \leqq \sum_{k=1}^{n} N_{\mu}\left(g_{k}\right) \leqq \sum_{k=1}^{\infty} N_{\mu}\left(g_{k}\right)
$$

Letting first $n \rightarrow \infty$ (by 1.13) and then $m \rightarrow \infty$ we obtain $N_{\mu}(f) \leqq \sum_{k=1}^{\infty} N_{\mu}\left(g_{k}\right)$ as required.

(5) Let $f_{m}$ be defined as in Definition 5 and for each $x \in T$ and each integer $m$ let $n(x, m)$ denote the least integer for which $f_{n(x, m)}(x) \geqq f_{m}(x)((m-1) / m)$.

If $X_{n m}=\{x: n(x, m) \leqq n\}$ then we have

$$
\left(\frac{m-1}{m}\right) V\left(f_{m} \mu, \mathfrak{A}\left[X_{n m}\right]\right) \leqq N_{\mu}\left(f_{n}\right) \leqq \sup _{k} N_{\mu}\left(f_{k}\right) .
$$

Again letting $n \rightarrow \infty$ (use 1.13) and then $m \rightarrow \infty$ we obtain $N_{\mu}(f) \leqq \sup _{k} N_{\mu}\left(f_{k}\right)$; as the opposite inequality is obvious this completes the proof. 
3. Integration. For a theory of integration we require more structure than has been necessary above; in particular we shall need sufficiently fine divisions of every elementary set.

Definition 6. A division system $(T, \mathfrak{A}, \mathfrak{B})$ is said to be a division space if every $\boldsymbol{S} \in \mathfrak{A}$ divides every elementary set.

There is a fully developed Riemann theory of integration in division spaces (Henstock [3], [4]; McShane [5]) which has the advantage over previous integrals of defining an integral for brick functions assuming their values in topological semigroups and which nonetheless possesses limit theorems of Lebesgue power.

We shall consider only the Lebesgue theory for these spaces and we are hence confined essentially to the real-valued case as in all Lebesgue theories.

Definition 7. A brick function $\mu$ on a division space $(T, \mathfrak{A}, \mathfrak{B})$ is said to be absolutely integrable (McShane [5, p. 15]) if for every $\varepsilon>0$ there is an $\boldsymbol{S} \in \mathfrak{P}$ so that

$$
\left(\mathfrak{D} \times \mathfrak{D}^{\prime}\right) \sum\left|\mu\left(I \cap I^{\prime}, x\right)-\mu\left(I^{\prime} \cap I, x^{\prime}\right)\right| \leqq \varepsilon
$$

for every pair of divisions $\mathfrak{D}$ and $\mathfrak{D}^{\prime}$ contained in $\boldsymbol{S}$.

If $\mu$ is a fixed brick function then the class of all real-valued functions $f$ on $T$ for which $f \mu$ is absolutely integrable and $N_{\mu}(|f|)<+\infty$ is denoted by $L(\mu)$; this collection is an ordered vector space under the usual pointwise definitions and we shall write $L_{+}(\mu)$ for the cone of nonnegative functions in $L(\mu)$.

THEOREM 3.1. Let $\mu$ be a nonnegative additive brick function on a division space $(T, \mathfrak{A}, \mathfrak{B})$ of type B. Then $L(\mu)$ is a Riesz space and $N_{\mu}$ is an additive functional on $L_{+}(\mu)$.

Proof. If $r: \boldsymbol{R}^{2} \rightarrow \boldsymbol{R}$ is any function satisfying the condition $\left|r\left(x_{1}, x_{2}\right)-r\left(y_{1}, y_{2}\right)\right|$ $\leqq M\left(\left|x_{1}-y_{1}\right|+\left|x_{2}-y_{2}\right|\right)$ for some positive constant $M$ then it is easy to show that for any pair of absolutely integrable brick functions $\mu_{1}$ and $\mu_{2}$, the brick function $r\left(\mu_{1}, \mu_{2}\right)$ is absolutely integrable. Thus if $f_{1}, f_{2} \in L(\mu)$ it follows readily that $\sup \left(f_{1}, f_{2}\right)$ and $\inf \left(f_{1}, f_{2}\right)$ both belong to $L(\mu)$.

Suppose $f_{1}$ and $f_{2}$ belong to $L_{+}(\mu)$ : since both $f_{1} \mu$ and $f_{2} \mu$ are additive we have by 1.12 that $N_{\mu}\left(f_{i}\right)=\sup _{E \in \mathbb{E}} V\left(f_{i} \mu, \mathfrak{A}(E)\right)(i=1,2)$. Choose $E_{0} \in \mathbb{E}$ so that

$$
V\left(f_{i} \mu, \mathfrak{A}\left(E_{0}\right)\right) \geqq N_{\mu}\left(f_{i}\right)-\varepsilon / 6 \quad(i=1,2)
$$

and choose $\boldsymbol{S} \in \mathfrak{A}$ so that

$$
\left(\mathfrak{D} \times \mathscr{D}^{\prime}\right) \sum\left|f_{2}(x) \mu\left(I \cap I^{\prime}, x\right)-f_{2}\left(x^{\prime}\right) \mu\left(I \cap I^{\prime}, x^{\prime}\right)\right| \leqq \varepsilon / 3
$$

for all $\mathfrak{D}, \mathfrak{D}^{\prime} \subseteq \boldsymbol{S}$.

Let $\mathfrak{D}$ and $\mathfrak{D}^{\prime}$ be divisions of $E_{0}$ chosen from $S$ so that

(D) $\sum f_{1}(x) \mu(I, x) \geqq V\left(f_{1} \mu, S\left(E_{0}\right)\right)-\varepsilon / 6$

and

$$
\left(\mathfrak{D}^{\prime}\right) \sum f_{2}\left(x^{\prime}\right) \mu\left(I^{\prime}, x^{\prime}\right) \geqq V\left(f_{2} \mu, S\left(E_{0}\right)\right)-\varepsilon / 6
$$


Then using the additivity of $\mu$ and the above computations

$$
\text { (D) } \begin{aligned}
\sum\left(f_{1}(x)+f_{2}(x)\right) \mu(I, x)= & (\mathfrak{D}) \sum f_{1}(x) \mu(I, x)+\left(\mathfrak{D}^{\prime}\right) \sum f_{2}\left(x^{\prime}\right) \mu\left(I^{\prime}, x^{\prime}\right) \\
& +\left(\mathfrak{D} \times \mathfrak{D}^{\prime}\right) \sum\left[f_{2}(x) \mu\left(I \cap I^{\prime}, x\right)-f_{2}\left(x^{\prime}\right) \mu\left(I \cap I^{\prime}, x^{\prime}\right)\right] \\
\geqq & N_{\mu}\left(f_{1}\right)+N_{\mu}\left(f_{2}\right)-\varepsilon .
\end{aligned}
$$

Hence $V\left(\left(f_{1}+f_{2}\right) \mu ; S\right) \geqq N_{\mu}\left(f_{1}\right)+N_{\mu}\left(f_{2}\right)-\varepsilon$ so that $N_{\mu}\left(f_{1}+f_{2}\right) \geqq N_{\mu}\left(f_{1}\right)+N_{\mu}\left(f_{2}\right)$. Since the opposite inequality is obvious it follows that $N_{\mu}$ is additive on $L_{+}(\mu)$ as required.

As a result of this theorem there is a unique linear extension of $N_{\mu}$ from $L_{+}(\mu)$ to the whole of the vector space $L(\mu)$ and we write this extension as $\int_{T} f d \mu(f \in L(\mu))$. Note that we have $\left|\int_{T} f d \mu\right| \leqq N_{\mu}(|f|)$ so that $f \rightarrow \int_{T} f d \mu$ is a continuous linear functional on the space $L(\mu)$ where this latter is equipped with the seminorm $f \rightarrow N_{\mu}(|f|)$.

Normally the Lebesgue theory now proceeds by forming the completion of the space $L(\mu)$ and by extending the integral $\int_{T} f d \mu$ by continuity to this enlarged space. The principal feature of this present theory is that this step is unnecessary; the space $L(\mu)$ is already complete and so the class of functions to be integrated is completely characterized by Definition 7 .

LEMMA 3.2. Let $\mu$ be a brick function on a decomposable division space. If $f_{n}$ is a sequence of functions in $L(\mu)$ and $N_{\mu}\left(\left|f_{n}-f_{m}\right|\right) \rightarrow 0$ as $n, m \rightarrow \infty$ then $f_{n}$ contains a subsequence which converges almost everywhere- $\mu$ *.

Proof. For every $\varepsilon>0$ define the set

$$
X_{m n}(\varepsilon)=\left\{x:\left|f_{n}(x)-f_{m}(x)\right|>\varepsilon\right\}
$$

so that

$$
\mu^{*}\left(X_{m n}(\varepsilon)\right) \leqq(1 / \varepsilon) V\left(\left(f_{n}-f_{m}\right) \mu, \mathfrak{2}\left[X_{m n}(\varepsilon)\right]\right) \leqq(1 / \varepsilon) N_{\mu}\left(\left|f_{n}-f_{m}\right|\right) .
$$

Choose an integer $n(\varepsilon)$ so that $N_{\mu}\left(\left|f_{n}-f_{m}\right|\right)<\varepsilon^{2}$ for every $m, n \geqq n(\varepsilon)$, and then for such $m, n$ we have $\mu^{*}\left(X_{m n}(\varepsilon)\right) \leqq \varepsilon$. Let $n_{1}<n_{2}<\cdots$ be an increasing sequence of integers satisfying $n_{k} \geqq n\left(1 / 2^{k}\right)$ for each $k$ and define the sets $X_{k}=X_{n_{k} n_{k+1}}\left(1 / 2^{k}\right)$, $Y_{k}=\bigcup_{i \geqq k} X_{i}$ and $Y_{0}=\bigcap_{i=1}^{\infty} Y_{i}$.

Then if $x \in T \backslash Y_{m}$ we have from the above that

$$
\left|f_{n_{k}}(x)-f_{n_{p}}(x)\right| \leqq \sum_{i=k}^{p} 1 / 2^{i} \leqq 1 / 2^{k-1} \text { for } p>k \geqq m .
$$

Thus the sequence $\left\{f_{n_{k}}(x)\right\}$ converges at every point of $T \backslash Y_{m}$ and so at every point of $T \backslash Y_{0}$. But

$$
\mu^{*}\left(Y_{0}\right) \leqq \mu^{*}\left(Y_{m}\right) \leqq \sum_{k \geqq m} \mu^{*}\left(X_{k}\right) \leqq \sum_{k \geqq m} 1 / 2^{k}=1 / 2^{m-1}
$$

for every integer $m$ so that $\mu^{*}\left(Y_{0}\right)=0$ and $\left\{f_{n_{k}}(x)\right\}$ converges almost everywhere- $\mu^{*}$ as required. 
THEOREM 3.3. Let $\mu$ be a nonnegative additive brick function on a decomposable division space $(T, \mathfrak{A}, \mathfrak{B})$ of type $\mathrm{B}$. If $f_{n}$ is a sequence of functions in $L(\mu)$ such that $N_{\mu}\left(\left|f_{n}-f_{m}\right|\right) \rightarrow 0$ as $n, m \rightarrow \infty$ then there exists a function $f$ in $L(\mu)$ such that $N_{\mu}\left(\left|f_{n}-f\right|\right) \rightarrow 0$ as $n \rightarrow \infty$.

Proof. Let $\left\{f_{n_{k}}\right\},\left\{Y_{m}\right\}$ and $Y_{0}$ be constructed from $\left\{f_{n}\right\}$ as in the proof of the lemma, and set $f(x)=\lim _{k \rightarrow \infty} f_{n_{k}}(x)\left(x \in T \backslash Y_{0}\right)$ and $f(x)=0\left(x \in Y_{0}\right)$.

Define the additive brick functions $\mu_{k}$ by setting $\mu_{k}(I, x)=\left(f_{n_{k}}(x)-f(x)\right) \mu(I, x)$ and note that $V\left(\mu_{k}-\mu_{k^{\prime}}, \mathfrak{X}\right) \rightarrow 0$ as $k, k^{\prime} \rightarrow \infty$. But for $X \subseteq T\left|\mu_{k}^{*}(X)-\mu_{k^{\prime}}^{*}(X)\right|$ $\leqq V\left(\mu_{k}-\mu_{k^{\prime}}, \mathfrak{X}[X]\right) \leqq V\left(\mu_{k}-\mu_{k^{\prime}}, \mathfrak{A}\right)$ so that $\lim _{k \rightarrow \infty} \mu_{k}^{*}(X)=\nu(X)$ exists uniformly for $X \subseteq T$ and it can be thus verified that $\nu$ is a measure on $T$ which inherits the property of Theorem 1.11 from the measures $\mu_{k}^{*}$.

Define the sets $A_{i j}=\left\{x:\left|f_{i}(x)\right| \geqq 1 / j\right\}$ and then $\mu^{*}\left(A_{i j}\right) \leqq j V\left(f_{i} \mu, \mathfrak{A}\right)<+\infty$; now from the construction of the sets $\left\{Y_{m}\right\}$ in the lemma

$$
\mu_{k}^{*}\left(A_{i j} \backslash Y_{m}\right) \leqq 1 / 2^{k-1} \mu^{*}\left(A_{i j}\right) \quad(k \geqq m)
$$

and hence $\nu\left(A_{i j} \backslash Y_{m}\right)=\lim _{k \rightarrow \infty} \mu_{k}^{*}\left(A_{i j} \mid Y_{m}\right)=0$ so that using the monotone property of $\nu$ and the fact that $\mu_{k}^{*}\left(Y_{0}\right)=0$ we also get

Finally if

$$
\nu\left(A_{i j}\right)=\nu\left(A_{i j} \backslash Y_{0}\right)=\lim _{m \rightarrow \infty} \nu\left(A_{i j} \backslash Y_{m}\right)=0 .
$$

$$
A_{0}=\left\{x: f_{i}(x) \neq 0, \text { some } i\right\}=\bigcup_{i=1}^{\infty} \bigcup_{j=1}^{\infty} A_{i j}
$$

then $\nu\left(A_{0}\right) \leqq \sum_{i, j} \nu\left(A_{i j}\right)=0$, so that

$$
\lim _{k \rightarrow \infty} V\left(\left(f_{n_{k}}-f\right) \mu, \mathfrak{i}\right)=\lim _{k \rightarrow \infty} \mu_{k}^{*}\left(A_{0}\right)=\nu\left(A_{0}\right)=0
$$

and hence $N_{\mu}\left(\left|f_{n_{k}}-f\right|\right) \rightarrow 0$ as $k \rightarrow \infty$. Standard arguments show that $N_{\mu}\left(\left|f_{n}-f\right|\right) \rightarrow 0$ as $n \rightarrow \infty$ as well.

It remains only to show that $f$ belongs to $L(\mu)$. Certainly $N_{\mu}(|f|) \leqq \sup _{n} N_{\mu}\left(\left|f_{n}\right|\right)$ $<+\infty$ so that we need only show that $f \mu$ is absolutely integrable.

If $\varepsilon>0$ choose $n_{0}$ so that $N_{\mu}\left(\left|f_{n_{0}}-f\right|\right)<\varepsilon / 6$ and choose $S \in \mathfrak{A}$ so that $V\left(\left(f_{n_{0}}-f\right) \mu, S\right)$ $\leqq N_{\mu}\left(\left|f_{n_{0}}-f\right|\right)+\varepsilon / 6$ and

$$
\left(\mathfrak{D} \times \mathscr{D}^{\prime}\right) \sum\left|f_{n_{0}}(x) \mu\left(I \cap I^{\prime}, x\right)-f_{n_{0}}\left(x^{\prime}\right) \mu\left(I^{\prime} \cap I, x^{\prime}\right)\right| \leqq \varepsilon / 3
$$

for all divisions $\mathfrak{D}, \mathfrak{D}^{\prime} \subseteq S$.

Now if $\mathscr{D}, \mathfrak{D}^{\prime} \subseteq S$ are arbitrary divisions we use the additivity of $\mu$ and the above computations to obtain

$$
\begin{aligned}
\left(\mathfrak{D} \times \mathfrak{D}^{\prime}\right) \sum \mid & f(x) \mu\left(I \cap I^{\prime}, x\right)-f\left(x^{\prime}\right) \mu\left(I \cap I^{\prime}, x^{\prime}\right) \mid \\
\leqq & \left(\mathfrak{D} \times \mathfrak{D}^{\prime}\right) \sum\left\{\left|f(x)-f_{n_{0}}(x)\right| \mu\left(I \cap I^{\prime}, x\right)+\left|f\left(x^{\prime}\right)-f_{n_{0}}\left(x^{\prime}\right)\right| \mu\left(I \cap I^{\prime}, x^{\prime}\right)\right\} \\
& +\left(\mathfrak{D} \times \mathfrak{D}^{\prime}\right) \sum\left|f_{n_{0}}(x) \mu\left(I \cap I^{\prime}, x\right)-f_{n_{0}}\left(x^{\prime}\right) \mu\left(I \cap I^{\prime}, x^{\prime}\right)\right| \\
\leqq & 2 V\left(\left(f-f_{n_{0}}\right) \mu, S\right)+\varepsilon / 3 \leqq \varepsilon
\end{aligned}
$$


so that $f \mu$ is absolutely integrable as required which completes the proof of the theorem.

It follows as well from the theorem that $\int_{T} f d \mu=\lim _{n \rightarrow \infty} \int_{T} f_{n} d \mu$; other limit theorems, in particular the Lebesgue monotone convergence theorem and the Lebesgue dominated convergence theorem follow now by standard arguments and it is quite evident that at this point the present theory can merge with the classical approaches.

In most cases the space $L(\mu)$ corresponds to the usual Lebesgue space associated with the measure $\mu^{*}$, but this is not true in general. We can however prove the following: if $\boldsymbol{M}(\mu)$ denotes the family of sets $X \subseteq T$ for which $\chi_{X} \in L(\mu)$ then (for additive brick functions $\mu$ on a decomposable division space of type B) $\boldsymbol{M}(\mu)$ is a semi-tribe, $\mu^{*}$ is countably additive on $\boldsymbol{M}(\mu)$, every function in $L(\mu)$ is measurable with respect to the semi-tribe $\boldsymbol{M}(\mu)$ and $\int_{T} f d \mu(f \in L(\mu))$ is equal to the measure theoretic integral of $f$ with respect to the measure $\mu^{*}$ on $\boldsymbol{M}(\mu)$. For spaces of type A the sets in $\boldsymbol{M}(\mu)$ are $\mu^{*}$-measurable but even if $X$ is $\mu^{*}$-measurable and $\mu^{*}(X)<+\infty$ it does not follow that $X \in \boldsymbol{M}(\mu)$; thus the measure theoretic integral may be stronger than the integral defined here.

\section{REFERENCES}

1. N. Dunford and J. T. Schwartz, Linear operators. I: General theory, Pure and Appl. Math., vol. 7, Interscience, New York, 1958. MR 22 \#3302.

2. R. Henstock, Theory of integration, Butterworths, London, 1963. MR 28 \#1274.

3. - Linear analysis, Butterworths, London, 1967.

4. - Generalized integrals of vector-valued functions, Proc. London Math. Soc. (3) 19 (1969), 509-536. MR 40 \#4420.

5. E. J. McShane, A Riemann-type integral that includes Lebesgue-Stieltjes, Bochner and stochastic integrals, Mem. Amer. Math. Soc. No. 88 (1969).

Simon Fraser University, Burnaby, B.C., Canada 